J. Lake Sci.(湖泊科学), 2008, 20(3): 344-350

http://www.jlakes.org. E-mail: jlakes@niglas.ac.cn

(C)2008 by Journal of Lake Sciences

\title{
食物浓度对模糊秀体溞生长和繁殖的影响*
}

\author{
尹 娟, 韩博平 ${ }^{* *}$ \\ (暨南大学水生生物研究所, 广州 510632)
}

摘 要: 模糊秀体溞是热带水体中枝角类的主要优势种, 它在竞争及对热带水体的适应方面的优势尚不清楚. 本文以模糊秀 体溞为研究对象, 观察不同食物浓度下其生长和繁殖的特点, 探讨模糊秀体泽成热带水体优势种的原因. 以蛋白核小球藻为 食物, 浓度梯度设为 $0.05 \mathrm{mg} / \mathrm{L} 、 0.1 \mathrm{mg} / \mathrm{L} 、 0.3 \mathrm{mg} / \mathrm{L} 、 0.6 \mathrm{mg} / \mathrm{L}$ 和 $1.0 \mathrm{mg} / \mathrm{L}$. 小球藻浓度为 $0.05 \mathrm{mg} / \mathrm{L}$ 的培养液中, 模糊秀体溞存活 率很低且未观察到繁殖现象. 其它 4 个浓度小球藻喂养的模糊秀体溞体长生长率和最大体长均随食物浓度的升高而增加, 最 大体长分别达到 $1.087 \mathrm{~mm} 、 1.125 \mathrm{~mm} 、 1.225 \mathrm{~mm} 、 1.313 \mathrm{~mm}$; 食物浓度对模糊秀体溞的胚胎发育时间, 平均寿命, 个体累计产 幼数无显著影响; 随着食物浓度的升高, 模糊秀体溞初次怀卵体长显著增加, 繁殖前期的历时相应缩短, 平均每胎产幼数增 加; 内禀增长率随食物浓度升高而增大, 达到 $0.6 \mathrm{mg} / \mathrm{L}$ 后比较稳定. 浓度为 $0.6 \mathrm{mg} / \mathrm{L}$ 时, 模糊秀体溞的净生殖率也达到最大值. $1.0 \mathrm{mg} / \mathrm{L}$ 组的世代时间最短，内禀增长率接近最大值. $0.6 \mathrm{mg} / \mathrm{L}$ 和 $1.0 \mathrm{mg} / \mathrm{L}$ 为模糊秀体溞生长比较适宜的食物浓度. 热带自然水 体中, 模糊秀体溞相对其它浮游动物个体小和怀卵率低的现象并非食物不足所致; 食物充足条件下的最大成体的个体大小与 野外样品接近，也说明自然水体中捕食并没有显著影响成体的个体大小. 模糊秀体溞体型较小、身体透明、行动敏捷，容易逃 避鱼类的捕食, 可能是模糊秀体溞能在食物丰富的热带水体中, 特别是透明度较低的富营养化湖泊中能够成为枝角类中优势 种的主要原因.

关键词: 模糊秀体溞; 食物浓度; 生长; 繁殖; 热带

\section{Effects of algal food concentration on the growth and reproduction of Diaphanosoma dubia}

YIN Juan \& HAN Boping

(Institute of Hydrobiology, Jinan University, Guangzhou 510632, P.R China)

Abstract: Diaphanosoma dubia is one of the dominant species in most tropical reservoirs, however, how it becomes dominant and whether food concentration plays a key role are unclear. In order to study the effects of algal food concentration on the growth and reproduction of D. dubia and to understand the possible causes for this small animal to be one of the dominant species in the tropical water bodies, the animals were fed by Chlorella pyrenoidosa as food with five organic carbon concentrations: $0.05 \mathrm{mg} / \mathrm{L}, 0.1 \mathrm{mg} / \mathrm{L}$, $0.3 \mathrm{mg} / \mathrm{L}, 0.6 \mathrm{mg} / \mathrm{L}$ and $1.0 \mathrm{mg} / \mathrm{L}$, respectively. The survival rate was quite low and no reproduction at the food concentration of $0.05 \mathrm{mg} / \mathrm{L}$. The somatic growth rate and longest body length increased with the organic carbon concentrations of Chlorella pyrenoidosa, the longest body length was $1.087 \mathrm{~mm}, 1.125 \mathrm{~mm}, 1.225 \mathrm{~mm}$ and $1.313 \mathrm{~mm}$, respectively. Food concentration showed no significant effect on embryonic and juvenile development periods, lifespan and the number of the cumulative eggs for each adult. The size at maturity and the mean number of eggs per brood both increased with the increasing organic carbon concentrations of Chlorella pyrenoidosa, but the duration of reproduction decreased. The intrinsic rate of increase increased with the increasing organic carbon concentrations of Chlorella pyrenoidosa. The net reproduction rate and the intrinsic rate of increase showed peak values at the food concentration of $0.6 \mathrm{mg} / \mathrm{L}$. Under the maximal food concentration of $1.0 \mathrm{mg} / \mathrm{L}$, the generation time was shortest and the intrinsic rate of increase was closed to the peak value. The proper concentrations for the growth of $D$. dubia were $0.6 \mathrm{mg} / \mathrm{L}$ and $1.0 \mathrm{mg} / \mathrm{L}$. The $D$. dubia is smaller than many species in cladocerans such as Daphnia galeata and has lower number of eggs per female. Its small body

* 国家自然科学基金(30670345)和教育部博士点基金(20050559004)联合资助. 2007-06-18 收稿; 2007-10-07 收修改稿. 尹 娟, 女, 1983 年生, 硕士研究生; E-mail: yinjuanzhi@163.com.

** 通讯作者; E-mail: tbphan@jnu.edu.cn. 
size and few eggs seems not be caused by low food concentration. The maximal sized animals cultured in the high food concentration is close to the animals found in natural water bodies, this means that predation doesn't significantly reduce its body size. Small size, transparent body and quickly moving enhance its advantages to dominate in tropical water bodies.

Keywords: Diaphanosoma dubia; food; growth; reproduction; tropics

秀体溞(Diaphanosoma) 是一种体型较小、身体透明、行动敏捷的枝角类. 其体长存在种间差异，同时 也受食物及环境等因素的影响而存在差异, 大部分种类的成体长度在 $0.4-2.5 \mathrm{~mm}$ 之间, 雌体体长一般都 大于同种雄体体长. 秀体溞是枝角类的重要种类, 在湖泊和水库中分布广泛, 是热带水体中重要的枝角 类优势种群. 根据 2000 年对广东省水库浮游动物组成结构的调查, 秀体溞是多数水库中枝角类的主要优 势种 ${ }^{[1]}$. 秀体溞种类较多, 在广东省水库中分布的主要种类有湛江甘村水库的模糊秀体溞(Diaphanosoma dubia)、珠海大镜山水库的短尾秀体溞(Diaphanosoma brachyurum)、从化流溪河水库的奥氏秀体溞 (Diaphanosoma orghidani transamurensis)等.

目前关于秀体溞的研究还存在许多需要深人研究的问题. 通常认为秀体溞适温范围狭窄, 每胎卵的 数目少, 导致出生率低, 在实验室不易进行连续培养观察 ${ }^{[2]}$, 国内外对其研究较少. 在种类的鉴定上, 由 于参考的分类标准不一致，导致了一些秀体溞种类的误定. 国内对秀体溞的研究中大部分仅限于其分布 情况, 而对其实验生态研究甚少, 黄祥飞 ${ }^{[2-3]}$ 对短尾秀体溞的发育和生长进行了研究, 并调查了 1983-1985 年间武汉东湖短尾秀体溞的的种群变动和生产量. 韩士群等 ${ }^{[4]}$ 研究了长肢秀体溞(Diaphanosoma leuchtenbergianum) (该种现已归名为短尾秀体溞)对富营养化水体藻类的生物操纵. Liu 等 ${ }^{[5]}$ 研究了 徐家河水库中秀体溞的垂直迁移情况和种群动态. Lemke 和 Benke $\mathrm{e}^{[6]}$ 观察了美国东南部一个小型湿地中短 尾秀体溞的生长和繁殖情况. Carmauze 等 ${ }^{[7]}$ 在研究 Chad 湖的浮游动物时, 对该湖的镰角秀体溞 (Diaphanosoma excisum) 的卵与胚胎及幼龄发育时间进行了培养和观察. Lopes 等 ${ }^{[8]}$ 以短尾秀体溞为材料 研究了农药残留对热带水生生态系统的影响. Pagano 等 ${ }^{[9]}$ 观察了镰角秀体溞的种群增长能力及影响因素. 这些研究都着重于温度、盐度和 $\mathrm{pH}$ 值等对秀体溞生长和繁殖的影响, 而食物对其影响则少有报道, Shrivastava 等 ${ }^{[10]}$ 以西里伯秀体溞(Diaphanosoma celebensis) 为材料, 研究了食物及食物浓度对其体长增

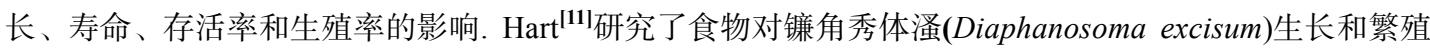
的影响. 为了解释秀体溞在热带小型富营养湖泊中个体小, 数量少且能成为优势种的主要原因, 本文以 从热带富营养化湖泊中分离出的模糊秀体溞为研究对象, 采用 5 个浓度的蛋白核小球藻对其进行培养, 通过观察其生长, 发育和繁殖情况, 分析食物浓度对模糊秀体溞生长和繁殖的影响, 并结合实验结果对 自然水体中模糊秀体溞如何获得种群优势的原因进行进一步地探讨.

\section{1 材料与方法}

\section{1 实验材料}

模糊秀体溞采自广州暨南大学明湖，从采集的浮游动物样品中分离出模糊秀体溞，以浓度为 $0.6 \mathrm{mg} / \mathrm{L}$ 的蛋白核小球藻为食物，经过多代孤䧳繁殖后，从中挑取龄期不超过 $24 \mathrm{~h}$, 活泼的新生幼体作为 实验材料.

实验室条件下培养的蛋白核小球藻, 直径约为 $4.1 \mu \mathrm{m}$, 小球藻的浓度用血球记数板记数(并根据单个 小球藻的含碳值换算成有机碳浓度), 每天计数一次.

模糊秀体溞培养液为一定浓度的小球藻藻液. 用 $0.45 \mu \mathrm{m}$ 玻璃纤维膜过滤明湖水, 将记数后的小球 藻用该过滤水进行稀释, 得到实验所需培养液. 培养液中蛋白核小球藻浓度分别为 $0.05 、 0.1 、 0.3 、 0.6$ 、 $1.0 \mathrm{mg} / \mathrm{L}$.

模糊秀体溞培养条件：温度 $23 \pm 1^{\circ} \mathrm{C}$ ，自然光照.

\section{2 实验方法}

挑取龄期不超过 $24 \mathrm{~h}$ 的新生溞体，单个培养于容积为 $50 \mathrm{ml}$ 的烧杯中, 烧杯中加人 $40 \mathrm{ml}$ 培养液, 培养液 中蛋白核小球藻浓度分别为 $0.05 、 0.1 、 0.3 、 0.6 、 1.0 \mathrm{mg} / \mathrm{L}$, 每个浓度设 15 个平行. 为保证较为稳定的培养 
液浓度, 实验期间每天更换培养液一次，同时观察死亡个体数，在解剖镜下测量存活个体大小; 待个体 发育成熟，开始怀卵时，同步记录首次怀卵时间、首次产幼时间、存活个体数、产幼量(由于孵育囊中的 卵数不易记数, 故假定所产之卵均能睬化, 而以产出之幼体数作为每窝的卵数); 新生个体移出, 不与母 体同养.

\section{3 数据分析}

本文数据用平均值 \pm 标准差表示, 用SPSS统计软件处理数据.

\section{2 实验结果}

\section{1 不同食物浓度下模糊秀体溞的繁殖参数}

在蛋白核小球藻浓度为 $0.05 \mathrm{mg} / \mathrm{L}$ 的培养液中, 模糊秀体溞死亡率很高且未观察到繁殖现象. 不同食 物浓度培养时模糊秀体掻的繁殖参数见表 1 . 统计分析结果显示, 同一温度, 光照条件下 $\left(23 \pm 1^{\circ} \mathrm{C}\right.$, 自然条 件光照, 下同), 食物的浓度与模糊秀体溞的胚胎发育时间、平均寿命、个体累计产幼数均无显著相关性 $(P>0.05)$.

食物的浓度对模糊秀体溞首次怀卵体长、繁殖前期历时、平均每胎产幼数均有显著影响 $(P<0.05)$, 随 着食物浓度的升高, 模糊秀体溞初次怀卵体长显著增加, 繁殖前期的历时显著缩短. 平均每胎产幼数的 总变化趋势是随浓度升高而增加, 在食物浓度为 $0.1 \mathrm{mg} / \mathrm{L}$ 时, 平均每胎产幼数明显小于其它三组, 食物浓 度为 $0.3 \mathrm{mg} / \mathrm{L}$ 和 $0.6 \mathrm{mg} / \mathrm{L}$ 时，平均每胎产幼数差别不大，增加趋势不明显，当浓度达到 $1.0 \mathrm{mg} / \mathrm{L}$ 时，平均每 胎产幼数显著增加.

通过曲线拟合，模糊秀体溞初次怀卵体长与食物浓度的关系符合方程 $Y=\mathrm{e}^{6.83-0.16 x}\left(r^{2}=0.984, n=15\right.$, $P=0.008)$; 模糊秀体溞繁殖前期的历时与食物浓度的关系符合指数方程 $Y=3.606 X^{0.346}\left(r^{2}=0.984, n=15\right.$, $P=0.008)$; 平均每胎产幼数与食物浓度的关系符合指数方程 $Y=4.868 X^{0.39}\left(r^{2}=0.987, n=15, P=0.006\right)$.

表1 不同食物浓度下模糊秀体溞的繁殖参数

Tab.1 Parameters of reproduction of D.dubia at 4 food concentrations

\begin{tabular}{lllll}
\hline \multirow{2}{*}{ 繁殖参数 } & \multicolumn{4}{c}{ 食物浓度 $(\mathrm{mg} / \mathrm{L})$} \\
\cline { 2 - 5 } & 0.1 & 0.3 & 0.6 & 1.0 \\
\hline 繁殖前期历时 $(\mathrm{d})$ & $8.17 \pm 0.84$ & $5.17 \pm 0.72$ & $4.50 \pm 0.52$ & $3.58 \pm 0.52$ \\
胚胎发育时间 $(\mathrm{d})$ & $3.33 \pm 0.49$ & $3.25 \pm 0.45$ & $3.00 \pm 0.60$ & $3.16 \pm 0.57$ \\
平均寿命 $(\mathrm{d})$ & $23.33 \pm 10.45$ & $21.58 \pm 7.31$ & $22.33 \pm 10.01$ & $17.92 \pm 10.64$ \\
首次怀卵体长 $(\mu \mathrm{m})$ & $793.75 \pm 6.52$ & $842.62 \pm 15.50$ & $876.04 \pm 15.50$ & $921.87 \pm 9.42$ \\
个体累计产幼数 & $9.41 \pm 7.77$ & $7.50 \pm 6.36$ & $14.75 \pm 13.43$ & $11.91 \pm 13.90$ \\
平均每胎产幼数 & $2.00 \pm 0.60$ & $3.08 \pm 0.66$ & $3.75 \pm 0.96$ & $5.08 \pm 1.08$ \\
\hline
\end{tabular}

\section{2 不同食物浓度下模糊秀体溞的存活率}

不同食物浓度条件下，模糊秀体溞的存活率开始下降的时间有所不同, $1.0 \mathrm{mg} / \mathrm{L}$ 组从第 $4 \mathrm{~d}$ 开始就出现较 多的个体死亡, $0.1 \mathrm{mg} / \mathrm{L}$ 组从第 $6 \mathrm{~d}, 0.3 \mathrm{mg} / \mathrm{L}$ 组从第 $7 \mathrm{~d}, 0.6 \mathrm{mg} / \mathrm{L}$ 组从第 $8 \mathrm{~d}$ 开始出现个体死亡. 模糊秀体溞的最 大存活时间以 $0.1 \mathrm{mg} / \mathrm{L}$ 最长, 达到了 $34 \mathrm{~d} .1 .0 \mathrm{mg} / \mathrm{L}$ 组和 $0.6 \mathrm{mg} / \mathrm{L}$ 组均为 $32 \mathrm{~d}, 0.3 \mathrm{mg} / \mathrm{L}$ 组最短, 为 $31 \mathrm{~d}$ (图 1 ).

\section{3 不同食物浓度下模糊秀体溞体长变化}

4 组蛋白核小球藻浓度条件下，模糊秀体溞体长增长速度受食物影响十分明显，随食物浓度的增加 而增加. 幼龄期模糊秀体溞体长生长速度都很快, $1.0 \mathrm{mg} / \mathrm{L}$ 组模糊秀体溞在第 $1-6 \mathrm{~d}$ 体长增长迅速, 每龄平 均体长增长达到了 $0.119 \mathrm{~mm}$; 在第 $7-20 \mathrm{~d}$ 增长速度有所降低, 每龄的体长增长约为 $0.025 \mathrm{~mm}$; 第 $20 \mathrm{~d}$ 以后, 模糊秀体溞体长生长速度显著降低，每龄体长增长仅为 $0.012 \mathrm{~mm}$; 在第 $32 \mathrm{~d}$ 达到的最大体长为 $1.313 \mathrm{~mm}$, 大于其它 3 个浓度条件下的最大体长.

$0.6 \mathrm{mg} / \mathrm{L}$ 组模糊秀体溞的体长增长速度略大于 $0.3 \mathrm{mg} / \mathrm{L}$ 组, 2 组在幼龄期的每龄平均体长增长分别为 
$0.093 \mathrm{~mm}$ 和 $0.096 \mathrm{~mm}$. 成龄前期, 两者体长增长速度十分接近. 但在成龄末期, $0.6 \mathrm{mg} / \mathrm{L}$ 组模糊秀体溞的 体长增长速度大于 $0.3 \mathrm{mg} / \mathrm{L}$ 组, $0.3 \mathrm{mg} / \mathrm{L}$ 组在末期生长速度十分缓慢, 近乎停止. $0.6 \mathrm{mg} / \mathrm{L}$ 组模糊秀体溞的 最大体长达到了 $1.225 \mathrm{~mm}$, 而 $0.3 \mathrm{mg} / \mathrm{L}$ 组为 $1.125 \mathrm{~mm}$.

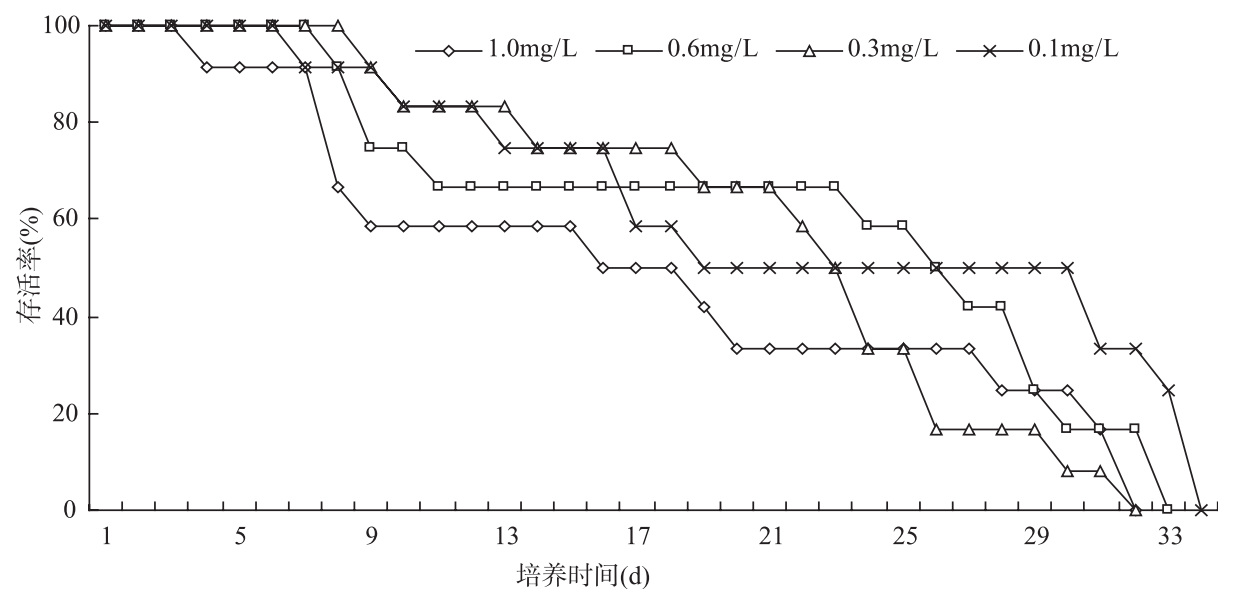

图1 不同食物浓度下模糊秀体溞的存活率

Fig.1 The survival rate of population of D.dubia at 4 food concentrations

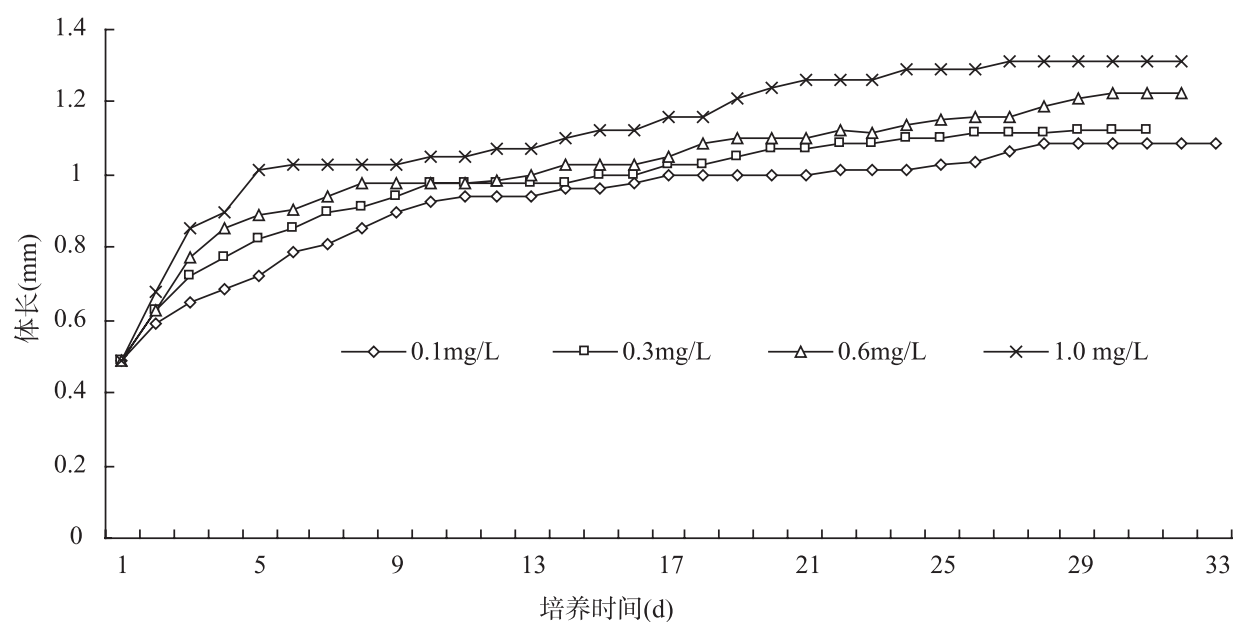

图2 不同食物浓度下模糊秀体溞的体长变化

Fig.2 Somatic growth of D.dubia at 4 food concentrations

$1 \mathrm{mg} / \mathrm{L}$ 组模糊秀体溞生长速度相对其它 3 个浓度组较为缓慢, 但其高速增长期持续时间长于其它浓度 组，达到了 $10 \mathrm{~d}$. 这个期间其每龄平均体长增长达到了 $0.0625 \mathrm{~mm}$ ，第 $11-28 \mathrm{~d}$ 每龄平均体长增长为 $0.018 \mathrm{~mm}$, 最大体长约为 $1.087 \mathrm{~mm}$.

\section{4 不同食物浓度下模糊秀体泽种群增长参数}

不同食物浓度条件下模糊秀体溞种群增长参数见表 2 , 随食物浓度升高, 模糊秀体溞的净生殖率分 别为 $9.40,8.42,14.9$ 和 11.9. 世代时间总的变化趋势是随食物浓度升高而缩短，在食物浓度为 $0.6 \mathrm{mg} / \mathrm{L}$ 时, 略有延长. 内禀增长率随食物浓度升高而增大, 达到 $0.6 \mathrm{mg} / \mathrm{L}$ 后比较稳定. 
表2 不同食物浓度下模糊秀体溞种群增长参数

Tab.2 Parameters of population growth of Diaphanosoma dubia at four food concentrations

\begin{tabular}{cccc}
\hline 食物浓度 $(\mathrm{mg} / \mathrm{L})$ & 净生殖率 $\left(R_{0}\right)$ & 世代时间 $G(\mathrm{~d})$ & 内禀增长率 $(r)$ \\
\hline 0.1 & 9.40 & 18.35 & 0.122 \\
0.3 & 8.42 & 16.42 & 0.129 \\
0.6 & 14.9 & 17.13 & 0.157 \\
1.0 & 11.9 & 15.85 & 0.156 \\
\hline
\end{tabular}

\section{3 讨论}

\section{1 食物浓度对模糊秀体泽生长的影响}

短尾秀体溞的生长一般分为三个时期：幼龄为高速生长期，成龄前期为中速生长期，后期为缓慢生 长期 ${ }^{[2]}$. 本实验中的模糊秀体溞在四个浓度的蛋白核小球藻条件下, 同样明显呈现出三个生长阶段. 这 是因为模糊秀体溞在幼龄期摄人的营养在转化为能量之后, 除去用于维持代谢的一部分, 剩余的能量主 要用于生长过程的消耗, 这表现在体长和体重的快速增加. 而到了成龄期, 除去用于维持代谢的一部分, 剩余的能量除了极少部份以体长、体重增加的生长方式消耗掉外, 大部分都用于生殖过程的消耗. 因此, 模糊秀体溞成龄期的生长率要远低于幼龄期的生长率.

同时，除去代谢后的能量在生长和生殖消耗间的比例关系决定了秀体泽的生长速度 ${ }^{[6]}$. 一般认为, 食物浓度越高体长生长率越高 ${ }^{[12]}$. 在较低食物浓度培养下的溞体将分配较少的能量用于生长和繁殖, 而 增加用于呼吸, 甲壳形成等维持代谢的能量 ${ }^{[13]}$. Daphnia hyalina $\times$ galeata 在饱和食物浓度下的个体最大 体长和体长生长率明显高于低食物浓度时 ${ }^{[14]}$. Daphnia obtusa 幼龄期的体长生长率随栅藻浓度的增加而 增加 ${ }^{[15]}$. 在相同的温度下, 高、中食物浓度下老年低额溞的各龄平均体长也明显大于低食物浓度下的平 均体长 ${ }^{[16]}$.

本实验中, 4 个浓度小球藻对模糊秀体溞的体长增长率有显著影响, 体长增长率和最大体长随食物浓 度的升高而增加, 最大体长也随食物浓度升高而增加, 模糊秀体溞与其它枝角类的体长增长有相似的特 点.

\section{2 食物浓度对模糊秀体泽发育和产卵量的影响}

一般情况下, 枝角类在较高食物浓度下比处于较低食物浓度时具有更优越的生殖性能 ${ }^{[17]}$. 食物浓度 的变化导致生殖性能的改变与物质能量的分配有关，在较低食物浓度培养下的溞体将分配较少的能量用 于生长和繁殖, 而增加用于呼吸, 甲壳形成等维持代谢的能量 ${ }^{[13]}$. 食物浓度越高, 首次怀卵时间越早 ${ }^{[14]}$. 随食物浓度增加,老年低额溞的首次怀卵时间呈提前趋势 ${ }^{[16]}$. 西里伯秀体溞在低浓度的球等鞭金藻 (Isochrysis galbana)和亚心形扁藻(Tetraselmis gracilis)食物条件下，首次怀卵时间则要早于相应的高浓度 食物条件下的首次怀卵时间 ${ }^{[10]}$.

本实验中, 食物浓度显著影响模糊秀体溞繁殖前期历时, 而对胚胎发育历时无显著影响. 随着食物 浓度的升高, 繁殖前期的历时相应缩短. 四个食物浓度条件下，模糊秀体溞的繁殖后期均较短，一般在 最后一次繁殖结束后 1-2d 内死亡.

食物浓度对模糊秀体溞首次怀卵体长有显著影响, 随着食物浓度的升高, 模糊秀体溞初次怀卵体长 显著增加．而 Daphnia hyalina $\times$ galeata 首次怀卵的个体大小不受食物浓度的影响 ${ }^{[14]}$.

模糊秀体溞的平均寿命在各浓度组中无显著差异. 但最大存活时间以 $0.1 \mathrm{mg} / \mathrm{L}$ 最长, 为 $34 \mathrm{~d} .1 .0 \mathrm{mg} / \mathrm{L}$ 组和 $0.6 \mathrm{mg} / \mathrm{L}$ 组均为 $32 \mathrm{~d}, 0.3 \mathrm{mg} / \mathrm{L}$ 组最短, 为 $31 \mathrm{~d}$.

食物的数量不仅影响模糊秀体溞个体的生长, 而且提供溞体生殖所必需的物质和能量. 产卵量是衡 量动物生命潜力的一个重要因素. 它一方面随种类, 年龄而异, 另一方面受外界环境的影响. 一般, 产卵 量与母体大小成正比 ${ }^{[18]}$. 在本实验当中, 平均每胎产幼数的总体变化趋势是随浓度升高而增加, 在 0.3-0.6mg/L的浓度范围内, 增加幅度较小. 模糊秀体溞平均每胎产幼数随浓度升高而增加的变化趋势与 在同一时间其体长随食物浓度升高而增加的趋势是一致的. 
模糊秀体溞是一种怀卵量较少的种群, 从不同食物浓度下培养的结果来看, 每胎卵数平均在1-6个 之间. 黄祥飞报道 ${ }^{[2]}$, 东湖短尾秀体溞每胎怀卵数平均在3.12-10.20个之间, 一般为 6-8个, 与本实验中观 察到的模糊秀体溞的怀卵量接近，可见，怀卵量在这两种秀体溞之间没有明显的种间差异.

\section{3 食物浓度对模糊秀体溞存活率和种群增长的影响}

$20^{\circ} \mathrm{C}$ 下不同食物浓度时老年低额溞在高浓度条件下的存活率低于低浓度 ${ }^{[16]}$. Nandini和Sarma ${ }^{[19]}$ 认为 随着食物密度增加而老年低额溞存活率和生殖率下降的原因可能是由于食物聚集的影响. 事实上, 在高 的食物浓度下, 某些枝角类为了清除食物对胸部附肢的障碍而花费更多的能量, 甚至使其处于饥饿状态 ${ }^{[20]}$.

在本实验中，高浓度食物条件下，模糊秀体溞在初始几天有很高的死亡率，而在低浓度下，模糊秀 体溞仍保持较高的存活率, 这与上述结果较为一致. 研究观察发现, 在较低的温度下, 生活在较高食物水 平下的老年低额溞(尤其是在幼龄期) 其身体更容易被自身产生的排泄物所细绕, 影响其胸部附肢活动 ${ }^{[16]}$. 本实验中也观察到类似的现象，这种不利现象的发生加速了模糊秀体溞的死亡.

不同品系 Daphnia longispina 的内禀增长率均随食物浓度的增加而增加 ${ }^{[21]}$. 过高食物浓度会导致溞 属呼吸作用加强, 净生殖率下降 ${ }^{[22]}$. 一般认为, 泽属在 $0.2-0.5 \mathrm{mg} / \mathrm{L}$ 食物浓度范围内, 净生殖率和内禀增

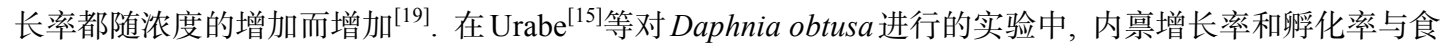
物浓度有相同的趋势, 且净生殖率也随食物浓度的增加而增加; 而 Boersma 等 ${ }^{[23]}$ 认为内禀增长率仅受食 物类型的影响, 与食物浓度无关. Daphnia laevis 的内禀增长率随小球藻浓度的增加而增加, 当食物浓度 达到 $4.69 \mathrm{mg} / \mathrm{L}$ 时，内禀增长率达到极值 ${ }^{[24]}$. Lemke 和 Benke ${ }^{[6]}$ 观察到美国东南部一个小型湿地中短尾秀体 溞在 $20^{\circ} \mathrm{C}$ 和 $25^{\circ} \mathrm{C}$, 自然食物条件时的内禀增长率分别为 0.15 和 0.32 .

本实验中，4组食物浓度条件下模糊秀体溞的平均寿命无显著差异，浓度为 $1.0 \mathrm{mg} / \mathrm{L}$ 时尽管初期死亡 率较高, 但由于较高的繁殖率, 使得该组仍具有较大的内禀增长率. 在浓度为 $0.6 \mathrm{mg} / \mathrm{L}$ 时, 该溞达到最大 净生殖率和内禀增长率. $0.6 \mathrm{mg} / \mathrm{L}$ 和 $1.0 \mathrm{mg} / \mathrm{L}$ 为模糊秀体溞种群增长比较适宜的食物浓度.

\section{4 模糊秀体泽在热带水体中成为枝角类优势种的原因}

暨南大学明湖中的模糊秀体溞体长接近于实验条件下单藻喂养的最大体长, 怀卵量也接近实验条件 下的最大值. 这说明该富营养化湖泊的自然食物对模糊秀体溞生长和繁殖并没有明显的抑制作用, 该自 然水体中鱼类捕食并没有降低模糊秀体溞最大的个体大小; 怀卵率极低的现象也不是由食物不够所导致, 而与该溞种的生活史与繁殖特性有关.

模糊秀体溞与其它大型浮游动物如盔形溞Daphnia galeata 相比, 其个体小, 且怀卵率极低. 实验室培 养条件下, 最大体长为 $1.313 \mathrm{~mm}$, 最大内禀增长率为 0.157 . 而盔形溞的体长和内毫增长率最大则分别达 到了 $2.63 \mathrm{~mm}$ 和 $0.42^{[25]}$. 相比之下, 模糊秀体溞在怀卵量方面并不具备成为优势种的最佳优势. 而在一些 贫营养的水库和湖泊中，模糊秀体溞的怀卵量更低的条件下，模糊秀体溞也能成为优势种，可见，食物 浓度并不是影响模糊秀体溞成为优势种的主要因素.

模糊秀体溞体型较小、身体透明、行动敏捷, 比其大型枝角类种类相比更容易逃避鱼类的捕食. $\mathrm{Liu}^{[26]}$ 报道了长江中下游水库中鱼类生物量对秀体溞丰度影响不明显的现象. 结合本实验结果, 秀体溞具有较 强的逃避捕食能力, 这有可能是模糊秀体溞能在食物丰富的热带水体中, 特别是透明度较低的富营养化 湖泊中能够成为枝角类中优势种的主要原因之一. 其它一些因素, 种间竞争也可能会影响到模糊秀体溞 的种群动态. 要完整地了解模糊秀体溞成为热带水体优势种的原因, 需要做进一步的研究和探讨.

致谢：本实验设计和秀体溞培养得到Dumont $\mathrm{H}$. 教授的指导，在此表示感谢！

\section{4 参考文献}

[1] 陈绵润, 赵帅营, 林秋奇等. 广东省枝角类组成特征的初步研究. 湖泊科学, 2007, 19(1): 77-86

[2] 黄祥飞. 短尾秀体溞的发育与生长. 水生生物学报, 1986, 10(1): 52-59.

[3] 黄祥飞. 武汉东湖短尾秀体溞的的种群变动和生产量. 生态学报, 1989, 9(2): 132-138.

[4] 韩士群, 严少华, 范成新等. 长肢秀体泽对富营养化水体藻类的生物操纵. 江苏农业学报, 2006, 22(1): 81-85.

[5] Liu ZW, Hu YH. Vertical distribution of the dominant planktonic crustaceans in a mesotrophic reservoir, Xujiahe reservoir 
(central China). Limnologica Ecology and Management of Inland Waters, 2001, 31(3): 171-178.

[6] Lemke AM, Benke AC. Growth and reproduction of three cladoceran species from a small wetland in the south-eastern U.S.A. Freshwater Biology, 2003, 48: 589-603.

[7] Carmouze JP, Durand JR, Lévêque C. LAKE CHAD ecology and productivity of a shallow tropical ecosystem. Kluwer Academic Publishers Group, Hague/Boston/Lancaster: 385-394

[8] Lopes I, Moreira-Santos M, da Silva E et al. In situ assays with tropical cladocerans to evaluate edge-of-field pesticide runoff toxicity. Chemosphere, 2007, 67(11): 2250-2256.

[9] Marc P, Saint-Jean L, Arfi R et al. Population growth capacities and regulatory factors in monospecific cultures of the cladocerans Moina micrura and Diaphanosoma excisum and the copepod. Thermocyclops decipiens from Côte d'Ivoire(West Africa). Aquatic Living Resources, 2000, 13(3): 163-172.

[10] Shrivastava Y, Mahambre GG, Achuthankutty CT et al. Parthenogenetic reproduction of Diaphanosoma celebensis (Crustacea: Cladocera). Effect of algae and algal density on survival, growth, life span and neonate production. Marine Biology, 1999, 135(4): 663-670.

[11] Hart RC. Experimental studies of food and suspended sed-iments effects on growth and reproduction of six planktonic cladocerans. Plankton Res, 1992, 14: 1425-1448.

[12] Giebelhausen B, Lampert W. Temperature reaction norms of Daphnia magna: the effect of food concentration. Freshwat Bio, 2001, 46: 281-289

[13] Stibor H, Lampet W. Estimating the size at maturity in field populations of Daphnta. Freshwater Biology, $1993,19: 240$.

[14] Dokster A, Vijverberg J. The effect of food and temperature regimes on life-history responses to fish kairomones in Daphnia hyalina $\times$ galeata. Hydrobiologia, 2001, 442: 207-214.

[15] Urabe J, Sterner RW. Contrasting effects of different types of resource depletion on life-history traits in Daphnia. Functional Ecology, 2001, 15: 165-174

[16] 邓道贵, 靳 英, 柳 明等. 温度和食物浓度对老年低额滍生长与生殖的影响. 水生生物学报, 2006, 30(3): 298-303.

[17] 杨四秀. 枝角类繁殖生物学研究概况. 水利渔业, 2004, 24(4): 12-14.

[18] 黄祥飞. 三种淡水枝角类的生物学研究. 海洋与湖沼, 1985, 16(3): 188-194

[19] Nandini S, Sarma SSS. Population growth of some genera of cladocerans (Cladocera) in relation to algal food (Chlorella vulgaris) levels. Hydrobiologia, 2003, 491: 211-219.

[24] Nandini S \& Sarma SSS. Lifetable demography of four cladoceran species in relation to algal food (Chlorella vulgaris) density. Hydrobiologia, 2000, 435: 117-126.

[20] Wang DL, Lai W. On growth and production of Simocephalus vetu- loides Sars. Journal of Fisheries China, 1990, 14(4): 304-312.

[21] Antunes SC, Castro BB, Goncalves F. Chronic responses of different clones of Daphnia longispina to different food levels. Acta Oecologica, 2003, 24: 325-332.

[22] Kennet GB, Gilbert JJ. Body size and food size in freshwater zooplankton. Ecology, 1984, 81: 6427-6431.

[23] Boersma M, Vijverberg J. Food effects on life history traits and seasonal dynamics of Ceriodaphnia pulchella. Freshwat Biology, 1996, 35: 25-34.

[25] 林 妚, 韩博平. 富营养化水体中自然食物对透明溞(Daphnia hyalina)生长与繁殖影响的初步实验. 生态科学, 2006, 25(2): 116-121.

[26] Liu ZW. Changes in abundance of the icefish Neosalanx pseudotaihuensis Zhang (Salangidae) and the impact on the zooplankton community of Xujiahe Reservoir, central China. Hydrobiologia, 2001, 445: 193-198. 